\title{
First Trimester Aneuploidy Screening Program for Preeclampsia Prediction in a Portuguese Obstetric Population
}

\author{
Cláudia Teixeira, ${ }^{1,2,3}$ Eduardo Tejera, ${ }^{3}$ Helena Martins, ${ }^{1}$ António Tomé Pereira, ${ }^{4}$ \\ Altamiro Costa-Pereira, ${ }^{2,5}$ and Irene Rebelo ${ }^{3,6}$ \\ ${ }^{1}$ Department of Pathology, Porto Hospital Center, 4099-001 Porto, Portugal \\ ${ }^{2}$ Department of Health Information and Decision Sciences (CIDES), Faculty of Medicine, University of Porto, 4200-450 Porto, Portugal \\ ${ }^{3}$ Institute for Molecular and Cell Biology (IBMC), University of Porto, 4150-180 Porto, Portugal \\ ${ }^{4}$ Department of Women, Porto Hospital Center, 4099-001 Porto, Portugal \\ ${ }^{5}$ Center for Research in Health Technologies and Information Systems (CINTESIS), Faculty of Medicine, University of Porto, \\ 4200-450 Porto, Portugal \\ ${ }^{6}$ Department of Biochemistry, Faculty of Pharmacy, University of Porto, 4050-313 Porto, Portugal
}

Correspondence should be addressed to Cláudia Teixeira; claudiateixeira@med.up.pt

Received 28 March 2014; Accepted 30 April 2014; Published 28 May 2014

Academic Editor: João Bernardes

Copyright (C) 2014 Cláudia Teixeira et al. This is an open access article distributed under the Creative Commons Attribution License, which permits unrestricted use, distribution, and reproduction in any medium, provided the original work is properly cited.

\begin{abstract}
Objective. To evaluate the performance of a first trimester aneuploidy screening program for preeclampsia (PE) prediction in a Portuguese obstetric population, when performed under routine clinical conditions. Materials and Methods. Retrospective cohort study of 5672 pregnant women who underwent routine first trimester aneuploidy screening in a Portuguese university hospital from January 2009 to June 2013. Logistic regression-based predictive models were developed for prediction of PE based on maternal characteristics, crown-rump length (CRL), nuchal translucency thickness (NT), and maternal serum levels of pregnancy-associated plasma protein-A (PAPP-A) and free beta-subunit of human chorionic gonadotropin (free $\beta$-hCG). Results. At a false-positive rate of 5/10\%, the detection rate for early-onset (EO-PE) and late-onset (LO-PE) PE was 31.4/45.7\% and 29.5/35.2\%, respectively. Although both forms of PE were associated with decreased PAPP-A, logistic regression analysis revealed significant contributions from maternal factors, free $\beta$-hCG, CRL, and NT, but not PAPP-A, for prediction of PE. Conclusion. Our findings support that both clinical forms of EO-PE and LO-PE can be predicted using a combination of maternal history and biomarkers assessed at first trimester aneuploidy screening. However, detection rates were modest, suggesting that models need to be improved with additional markers not included in the current aneuploidy screening programs.
\end{abstract}

\section{Introduction}

Preeclampsia (PE) is a prevalent clinical entity in pregnancy, which is responsible for substantial maternal-fetal morbidity and mortality [1-4]. Prediction of PE could offer a window of opportunity for intervention during pregnancy, making it potentially possible to prevent adverse obstetric and neonatal outcomes.

In the United Kingdom, the National Institute for Health and Clinical Excellence (NICE) has delivered clinical guidelines recommending the evaluation of maternal risk factors for PE at the first prenatal visit for all pregnant women [5].
However, screening for PE based only on maternal history has shown to be insufficient [6].

In this context, measurement in early pregnancy of a variety of markers implicated in the pathophysiology of PE has been proposed to predict its development. These included tests for aneuploidy screening, renal and endothelial dysfunction, oxidative stress, and fetal-derived products [7]. Because any single biomarker is unlikely to be effective in prediction of the onset of a disorder as heterogeneous as PE, it is under investigation which combinations of tests, such as ultrasound and serum markers, would raise the effectiveness of history and physical-based screening [7]. 


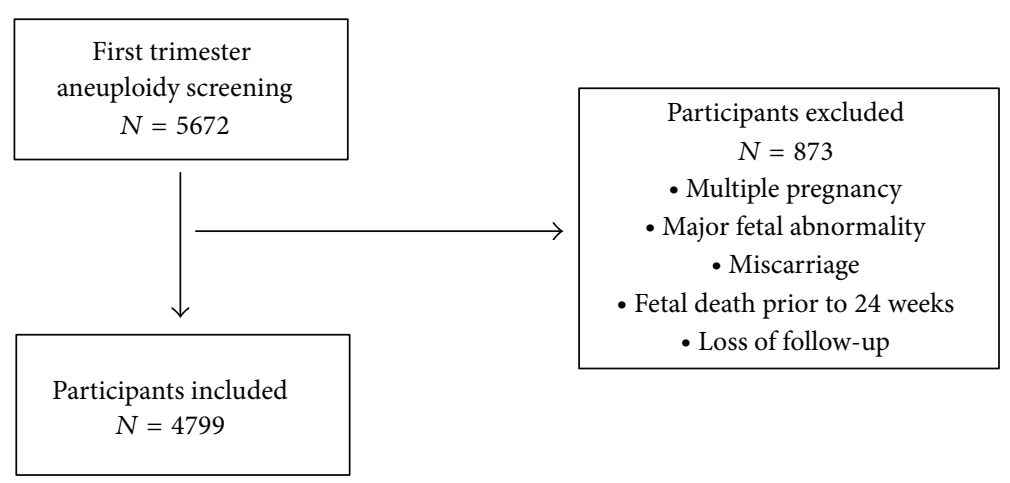

FIGURE 1: Flow chart of the study population.

The accuracy of models for PE prediction, either maternal history-based or combined with biomarkers, is unknown in the Portuguese population. Therefore, we aim to evaluate the performance of a first trimester aneuploidy screening program for preeclampsia prediction in a Portuguese obstetric population, when performed under routine clinical conditions.

\section{Materials and Methods}

2.1. Design, Setting, and Participants. This is a retrospective cohort study of 5672 pregnant women who underwent routine first trimester aneuploidy screening in a Portuguese university hospital (Centro Hospitalar do Porto) from January 2009 to June 2013. All singleton pregnancies between 9 weeks and 0 days and 13 weeks and 6 days of gestation were considered for inclusion in the study. Cases of multiple pregnancy, major fetal chromosomal or structural abnormalities, miscarriage, fetal death prior to 24 weeks, or loss of follow-up were excluded $(n=873)$ (Figure 1$)$. All participants were followed from first trimester combined aneuploidy screening until delivery. The study protocol has been approved by the local ethics committee and institutional review boards.

2.2. Maternal Evaluation. Combined screening for aneuploidies was performed between 9 weeks and 0 days and 13 weeks and 6 days of gestation $(n=4799)$. Participants were asked to provide information on age, ethnicity, method of conception, weight, smoking status, chronic conditions, parity and previous pregnancy complications. Blood samples were collected and maternal serum pregnancy-associated plasma protein-A (PAPP-A) and free beta-subunit of human chorionic gonadotropin (free $\beta$-hCG) were measured using routine automated analyzers. During the study period, the analytical platform used by the clinical chemistry laboratory for measuring these biochemical markers was changed from IMMULITE 2000 system $(n=1634)$ to DELFIA XPRESS analyzer $(n=3165)$; this event was taken into consideration in the statistical analysis of data. An ultrasound examination was performed between 11 weeks and 0 days and 13 weeks and 6 days of gestation, including the measurement of crownrump length (CRL) and nuchal translucency thickness (NT); gestational age was estimated on the basis of CRL measurements. These clinical data were systematically collected into an integrated electronic form in order to perform combined first trimester risk assessment.

2.3. Outcome Measures. Data on pregnancy outcome were collected from maternal and pediatric records. PE cases were defined by the new onset of hypertension $(>140 / 90 \mathrm{mmHg}$ ) developed after 20 weeks of gestation in a woman with previously normal blood pressure, associated by coexisting significant proteinuria, according to the definition of the American College of Obstetricians and Gynecologists (ACOG) [3]. Chronic hypertension cases were defined as known high blood pressure before conception or new onset of hypertension before 20 weeks of gestation [3]. In the cases in which $\mathrm{PE}$ was superimposed on chronic hypertension, there was significant proteinuria development after 20 weeks of gestation in women with known chronic hypertension [3]. Cases of new onset of hypertension after 20 weeks of gestation in the absence of accompanying proteinuria were considered as gestational hypertension [3]. These outcome diagnoses were made by the treating physician and registered in maternal records at hospital discharge. Preeclampsia cases were classified as early-onset (EO-PE) or late-onset (LO-PE), depending on when findings first become apparent, before or after 34 weeks of gestation. We also included obstetric and neonatal outcomes in our analysis, such as gestational age at delivery, delivery by cesarean section, stillbirth occurrence, and birth weight. The adopted definition of low birth weight (LBW) was birth weight below 2500 grams.

2.4. Statistical Analysis. A descriptive analysis of maternal characteristics was conducted, separating the unaffected group from the women affected by preeclampsia according to their PE status, as described in the previous section. The maternal weight, PAPP-A, and $\beta$-hCG were expressed as multiples of the median (MoM) and log transformed for logistic regression analysis. Considering that two distinct analytical platforms were used for PAPP-A and $\beta$-hCG measurement, we adjusted these differences by performing the MoM transformation with the median values of the samples of both assays. The MoM values distribution obtained by 
this procedure were not statistically different when compared by analytical method using ANOVA analysis. We performed Mann-Whitney $U$ Test and Pearson $\chi^{2}$ for single categorical and quantitative variables analysis across groups. Moreover, the Kruskal-Wallis test was also used to compare the quantitative variables across multiples groups with subsequent pairwise analysis.

ROC (Receiver Operating Characteristic) analysis was performed to evaluate models performances and estimate predictive values, which were presented as the estimated detection rate (DR) at fixed false-positive rate (FPR) of 5\% and $10 \%$. All the binomial logistic models were obtained using stepwise backward algorithm for variable selection and a $P$ value cutoff of 0.05 . We also presented the Nagelkerke $R^{2}$ for each model.

The statistical software package SPSS 21.0 [8] was used for data analyses.

\section{Results and Discussion}

3.1. Results. First trimester aneuploidy screening was carried out in 5672 pregnant women between January 2009 and June 2013. We excluded 873 cases because of missing outcome data $(n=715)$ or pregnancies resulting in miscarriage, fetal death prior to 24 weeks, or major fetal chromosomal or structural abnormalities $(n=158)$.

In the remaining 4799 cases, 140 developed PE (2.9\%) and 4659 were pregnancies unaffected by PE. In the PE group, 35 (25\%) developed early-onset PE and 105 (75\%) developed late-onset PE. Biomarkers included in first trimester combined aneuploidy screening were available in all cases. A descriptive analysis of maternal characteristics, aneuploidy screening biomarkers results, and pregnancy outcomes is presented in Table 1.

In the PE group, compared to unaffected pregnancies, there was a higher median maternal age and weight as well as a higher prevalence of nulliparous women and history of chronic hypertension or diabetes mellitus. There were no significant differences in maternal ethnic origin, smoking habits, type of conception and infant gender between groups. In both the EO-PE and LO-PE groups PAPP-A were lower compared to unaffected pregnancies; there were no significant differences in free $\beta$-hCG, CRL, and NT. Median gestational age at delivery was lower in PE group compared to unaffected pregnancies and in EO-PE group compared to LO-PE group. Delivery by cesarean section was carried out in $70.5 \%$ of $\mathrm{PE}$ cases compared to $34.1 \%$ in pregnancies unaffected by PE. There was a lower median birth weight in $\mathrm{PE}$ group compared to unaffected pregnancies and in EOPE group compared to LO-PE group, as well as a higher prevalence of LBW.

Although EO-PE and LO-PE were associated with decreased PAPP-A (0.93 MoM and 0.85 MoM), logistic regression analysis demonstrated that there were significant contributions from maternal factors, free $\beta$-hCG, CRL and NT, but not PAPP-A, for prediction of PE (Table 2). The $R^{2}$ values obtained were $10.0 \%, 9.4 \%$, and $10.3 \%$ for PE, EO-PE and LO-PE, respectively. As expected, maternal history of

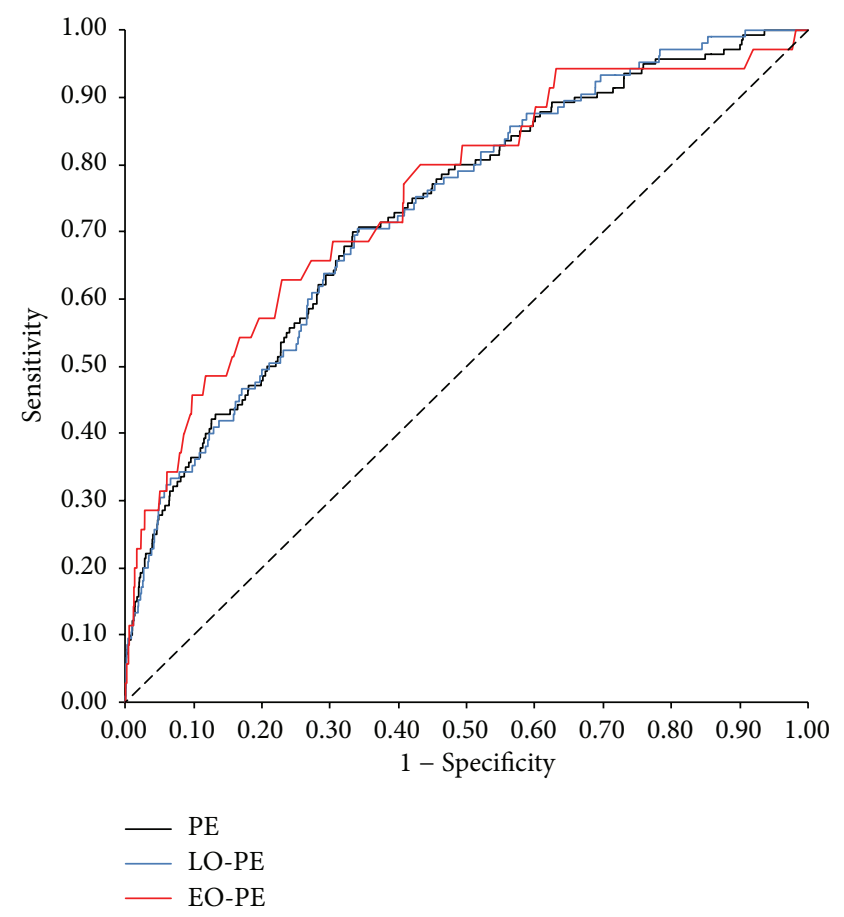

FIgURE 2: ROC curves of logistic regression models for prediction of PE, EO-PE, and LO-PE by maternal characteristics and aneuploidy screening biomarkers. PE, preeclampsia; EO-PE, early-onset preeclampsia; LO-PE, late-onset preeclampsia.

$\mathrm{PE}$, chronic hypertension, or diabetes mellitus contributed to the increase of the risk of PE, even when the last was not found significant for early expression of the disease. Similarly, higher maternal age and weight as well as nulliparous condition were also significant risk factors for PE.

Our logistic regression models for PE prediction estimate that $27.9 \%, 31.4 \%$, and $29.5 \%$ of PE, EO-PE, and LO-PE cases, respectively, could be detected with a $5 \%$ false-positive rate (Table 3). The model for EO-PE prediction presented the best performance (Figure 2), with detection rates of $31.4 \%$ and $45.7 \%$ at false-positive rates of $5 \%$ and $10 \%$.

A univariate analysis of PAPP-A and free $\beta$-hCG according to fetal weight at delivery shows lower median PAPPA in LBW group but no significant differences of free $\beta$ hCG (Figure 3). Moreover, when we considered a logistic regression model for LBW, including all the previous variables, the final obtained model is $1.886-1.013$ (if chronic hypertension $)+0.658$ (if caucasian $)-0.570$ (if smoker $)+$ 0.196 (if multiparous) + $1.155 *$ PAPP-A (MoM, Log) + $3.381 *$ Maternal Weight (MoM, Log). This suggests that, unlike in PE prediction models, PAPP-A is related to LBW when other variables are included in a logistic regression model.

3.2. Discussion. Our cohort study of 4799 pregnant women who underwent routine first trimester aneuploidy screening in a Portuguese university hospital found a prevalence of PE of $2.9 \%$, which is consistent with the reported epidemiologic data available in the literature $[1-4,9-11]$. 
TABLE 1: Demographic characteristics of the study population.

\begin{tabular}{|c|c|c|c|c|}
\hline Variable & $\begin{array}{l}\text { Unaffected pregnancy } \\
\qquad(n=4659)\end{array}$ & $\operatorname{PE}(n=140)$ & EO-PE $(n=35)$ & LO-PE $(n=105)$ \\
\hline Maternal age, years, median (IQR) ${ }^{* a}$ & $29.9(25.8-33.0)$ & $31.0(27.7-33.6)$ & $30.0(25.0-34.9)$ & $31.0(28.0-33.0)$ \\
\hline \multicolumn{5}{|l|}{ Maternal weight, median (IQR) } \\
\hline $\mathrm{Kg}^{* \mathrm{a}}$ & $63.5(57.0-72.0)$ & $70.0(60.9-82)$ & $73.0(64.5-82.0)$ & $69.3(60.6-83.4)$ \\
\hline $\mathrm{MoM}^{* \mathrm{a}}$ & $0.99(0.89-1.13)$ & $1.10(0.95-1.28)$ & $1.14(1.01-1.28)$ & $1.07(0.94-1.30)$ \\
\hline \multicolumn{5}{|l|}{ Ethnicity, $n(\%)$} \\
\hline White & $4529(97.2)$ & $138(98.6)$ & $34(97.1)$ & $104(99.0)$ \\
\hline Black & $82(1.8)$ & $1(0.7)$ & $1(2.9)$ & $0(0.0)$ \\
\hline Other & $48(1.0)$ & $1(0.7)$ & $0(0.0)$ & $1(1.0)$ \\
\hline Nulliparous, $n(\%)^{\mathrm{b}}$ & $2843(61.0)$ & $98(70.0)$ & $27(77.1)$ & $71(67.6)$ \\
\hline \multicolumn{5}{|l|}{ Medical history, $n(\%)$} \\
\hline Chronic hypertension $^{\mathrm{b}}$ & $104(2.2)$ & $12(8.6)$ & $5(14.3)$ & $7(6.7)$ \\
\hline Renal disease & $5(0.1)$ & $1(0.7)$ & $1(2.9)$ & $0(0.0)$ \\
\hline Diabetes mellitus $^{\mathrm{b}}$ & $47(1.0)$ & $9(6.4)$ & $1(2.9)$ & $8(7.6)$ \\
\hline Smoking during pregnancy, $n(\%)$ & $975(20.9)$ & $21(15.0)$ & $6(17.1)$ & $15(14.3)$ \\
\hline Spontaneous conception, $n(\%)$ & $4486(96.3)$ & $130(92.9)$ & $33(94.3)$ & $97(92.4)$ \\
\hline \multicolumn{5}{|l|}{ Ultrasound markers, median (IQR) } \\
\hline $\mathrm{CRL}, \mathrm{mm}$ & $62.9(56-70)$ & $64.5(58-70)$ & $63.2(56-68.9)$ & $65.0(59-70.5)$ \\
\hline $\mathrm{NT}, \mathrm{mm}$ & $1.5(1.2-1.8)$ & $1.5(1.2-1.8)$ & $1.5(1.2-1.9)$ & $1.5(1.1-1.8)$ \\
\hline \multicolumn{5}{|l|}{ Maternal serum, median (IQR) } \\
\hline PAPP-A, MoM ${ }^{* a}$ & $1.01(0.63-1.60)$ & $0.85(0.56-1.35)$ & $0.93(0.33-1.39)$ & $0.85(0.58-1.33)$ \\
\hline Free $\beta$-hCG, MoM & $1.00(0.66-1.54)$ & $1.10(0.66-1.64)$ & $0.93(0.53-1.38)$ & $1.17(0.70-1.76)$ \\
\hline \multicolumn{5}{|l|}{ Obstetric outcomes } \\
\hline Gestational hypertension, $n(\%)$ & $57(1.2)$ & $0(0.0)$ & $0(0.0)$ & $0(0.0)$ \\
\hline Cesarean section, $n(\%)^{\mathrm{b}}$ & $1589(34.1)$ & $98(70.5)$ & $31(88.6)$ & $67(64.4)$ \\
\hline Gestational age at delivery, weeks, median (IQR) $)^{* a}$ & $39(38-40)$ & $37(35-38)$ & $34(29-36)$ & $37(36-39)$ \\
\hline \multicolumn{5}{|l|}{ Neonatal outcomes } \\
\hline Male, $n(\%)$ & $2367(50.8)$ & $67(48.2)$ & $19(54.3)$ & $48(46.2)$ \\
\hline Stillbirth, $n(\%)$ & $14(0.3)$ & $2(1.4)$ & $2(5.7)$ & $0(0.0)$ \\
\hline Birth weight, g, median (IQR) ${ }^{* a}$ & $3165(2873-3440)$ & $2670(2150-3055)$ & $1910(1050-2440)$ & $2830(2481-3158)$ \\
\hline LBW, $n(\%)^{\mathrm{b}}$ & $354(7.6)$ & $55(39.6)$ & $27(77.1)$ & $28(26.9)$ \\
\hline
\end{tabular}

PE: preeclampsia; EO-PE: early-onset preeclampsia; LO-PE: late-onset preeclampsia; IQR: interquartile range; CRL: crown-rump length; NT: nuchal translucency thickness; $\beta$-hCG: beta-subunit of human chorionic gonadotropin; PAPP-A: pregnancy-associated plasma protein-A; MoM: multiple of the median; LBW: low birth weight. Significant comparisons between unaffected pregnancies and preeclampsia cases $(P<0.05)$ using: ${ }^{*}$ Mann-Whitney $U$ test, ${ }^{\mathrm{a}}$ Kruskal-Wallis test, and ${ }^{\mathrm{b}}$ Pearson $\chi^{2}$.

Our study provides evidence that both clinical forms of EO-PE and LO-PE can be predicted using a combination of maternal history and biomarkers assessed at first trimester aneuploidy screening, in agreement with previous publications [9-15]. As expected, regression models applied for prediction of the two forms of PE differed regarding which variables were included and performance achieved for each clinical form. However, these detection rates were modest, suggesting that models need to be improved with new information.

At false-positive rates of $5 \%$ and $10 \%$, the detection rates for EO-PE were $31.4 \%$ and $45.7 \%$, respectively, which are close to similar reported models $[10,12,15]$. Though EO-PE prediction presented a better performance compared to LO$\mathrm{PE}$, we must notice that its regression model only includes maternal clinical data, excluding biochemical and ultrasound markers. On the other hand, for LO-PE and overall PE prediction, logistic regression analysis revealed significant contributions from maternal factors, free $\beta$-hCG, CRL, and NT, but not from PAPP-A.

Although EO-PE and LO-PE were associated with decreased PAPP-A in the univariate analysis, this biomarker was not included in any of the logistic models for PE prediction. This could mean that the inclusion of PAPP-A in the models did not add further significant information to the one already provided by the others variables combined, hence the potential added value of PAPP-A measurement could be virtually negligible when used in combination with other biomarkers. These results suggest that a combination of free $\beta$-hCG, NT, and CRL could be more useful in PE prediction 
TABLE 2: Logistic regression models for prediction of PE, EO-PE and LO-PE by maternal characteristics and aneuploidy screening biomarkers.

\begin{tabular}{|c|c|c|c|c|c|c|}
\hline \multirow{2}{*}{ Variable } & \multicolumn{2}{|c|}{$\mathrm{PE}$} & \multicolumn{2}{|c|}{ EO-PE } & \multicolumn{2}{|c|}{ LO-PE } \\
\hline & $B$ & $P$-value & $B$ & $P$-value & $B$ & $P$-value \\
\hline Chronic hypertension [if true] & 0.870 & 0.014 & 1.519 & 0.004 & 0.050 & 0.012 \\
\hline Diabetes mellitus [if true] & 1.428 & 0.000 & & & 1.649 & 0.000 \\
\hline Parity [if multiparous] & -0.787 & 0.000 & -1.201 & 0.008 & -0.623 & 0.008 \\
\hline History of PE [if true] & 3.952 & 0.000 & 3.201 & 0.001 & 3.668 & 0.000 \\
\hline Maternal age & 0.039 & 0.026 & & & & \\
\hline Maternal weight (MoM, log) & 6.159 & 0.000 & 7.108 & 0.000 & 5.834 & 0.000 \\
\hline CRL & 0.027 & 0.010 & & & 0.036 & 0.003 \\
\hline NT & -0.483 & 0.036 & & & -0.592 & 0.026 \\
\hline Free $\beta$-hCG $(\mathrm{MoM}, \log )$ & 0.766 & 0.018 & & & 1.046 & 0.004 \\
\hline Constant & -5.723 & 0.000 & -4.951 & 0.000 & -8.248 & 0.000 \\
\hline
\end{tabular}

Note: Missing values or any variable not included in the table indicate that those variables were not selected for the final regression models by absence of statistical significance. PE: preeclampsia; EO-PE: early-onset preeclampsia; LO-PE: late-onset preeclampsia.

TABLE 3: Detection rates and ROC results of logistic regression models for prediction of PE, EO-PE, and LO-PE by maternal characteristics and aneuploidy screening biomarkers.

\begin{tabular}{lccc}
\hline Variable & ROC AUC & DR $(\mathrm{FPR}=5 \%)$ & DR $(\mathrm{FPR}=10 \%)$ \\
\hline PE & 0.732 & 27.9 & 36.4 \\
EO-PE & 0.754 & 31.4 & 45.7 \\
LO-PE & 0.734 & 29.5 & 35.2 \\
\hline
\end{tabular}

PE: preeclampsia; EO-PE: early-onset preeclampsia; LO-PE: late-onset preeclampsia; AUC: area under the curve; DR: detection rate; FPR: falsepositive rate.

than PAPP-A alone. Our findings are supported by other studies $[11,16,17]$ that also found a significant association between PE and PAPP-A that lost its significance when combined with others biomarkers and therefore did not contribute to PE prediction. Moreover, PAPP-A was significantly related to LBW, unlike free $\beta$-hCG, NT, and CRL; previous publications have shown inconsistent results regarding the association of birth weight with these biomarkers, as some studies reported a significant correlation $[18,19]$ and others did not $[14,20,21]$.

Our study presents some limitations related to its retrospective nature. First, serum measurement of PAPP-A and $\beta$ hCG was not performed by the same assay method for all participants due to change of the analytical platform used by the clinical chemistry laboratory during the study period; however, this event was taken into consideration in the statistical analysis of data. Additionally, although several studies [9-11, 15] have shown that mean arterial pressure (MAP) is an important predictive variable for PE, data on maternal arterial pressure at first trimester screening were not available in clinical records. Furthermore, diagnosis of PE cases was made by the treating physician, which could constitute a potential source of bias. Nevertheless, it is the first study of its kind conducted under routine clinical conditions in a Portuguese obstetric population, reflecting the reality of nearly five years of performing a first trimester prenatal screening program.

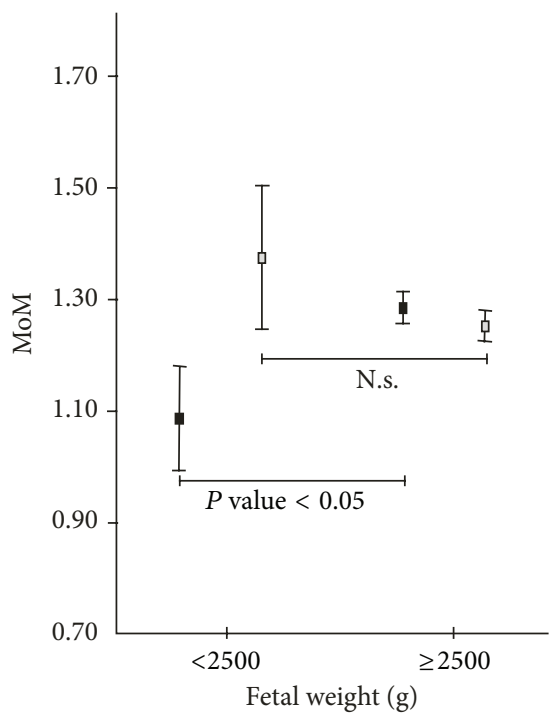

- PAPP-A

口 $\beta$-hCG

FIgURE 3: Variation of PAPP-A and $\beta$-hCG according to fetal weight at delivery (LBW).

As the traditional approach for PE screening based only on maternal demographic characteristics and medical history has shown to be insufficient [6], it is under investigation which combinations of markers would improve the performance of history-based screening. Ideal markers for PE screening should be easily measured and integrated within routine testing currently used as a part of prenatal screening. Furthermore, it would be helpful to integrate PE screening into existing analytical platforms to reduce costs, equipment, and human resources. Moreover, those markers should predict the risk in the first trimester of pregnancy, thus creating a wide window of opportunity to implement preventive or prophylactic treatment strategies which may facilitate normal placental development [22]. In this 
regard, biomarkers measured concurrently with testing for aneuploidies meet these requirements.

This study was conducted in a large unselected population under routine clinical care conditions, which supports the idea that screening for $\mathrm{PE}$ is feasible in obstetric populations with low a priori risk. However, our results suggest that the single inclusion of biomarkers currently used for aneuploidy screening in the prediction models for PE cannot achieve satisfactory detection rates and predictive values. Nevertheless, first trimester combined aneuploidy screening could be improved by inclusion of other biomarkers implicated in the pathophysiology of PE. Recent evidence suggests that serum placental growth factor (PlGF) and uterine artery pulsatility index (UtA Doppler) can be successfully included in preeclampsia prediction models with promising results $[9-13,15]$. Although those results may be encouraging, it is difficult to achieve generalizable conclusions and standardized cut-points at specific gestational ages due to divergent study designs, population characteristics, and statistical approaches. Therefore, performance of PE screening should be validated in further large prospective studies.

Although the performance of such approach in Portuguese population is unknown, we believe that screening for PE could be successfully incorporated into routine prenatal care for assessment of patient-specific risk for PE and therefore offer a window of opportunity for intervention in early pregnancy.

\section{Conclusion}

Our findings support that both clinical forms of EO-PE and LO-PE can be predicted using a combination of maternal history and biomarkers assessed at first trimester aneuploidy screening. However, detection rates were modest, suggesting that the models need to be improved with additional markers not included in current aneuploidy screening programs.

\section{Conflict of Interests}

The authors declare that there is no conflict of interests regarding the publication of this paper.

\section{References}

[1] WHO, World Health Report 2005: Make Every Mother and Child Count, World Health Organization, Geneva, Switzerland, 2005.

[2] L. Duley, "The global impact of pre-eclampsia and eclampsia," Seminars in Perinatology, vol. 33, no. 3, pp. 130-137, 2009.

[3] The American College of Obstetricians and Gynecologists, "Hypertension in pregnancy. Report of the American College of Obstetricians and Gynecologists' task force on hypertension in pregnancy," Obstetrics \& Gynecology, vol. 122, no. 5, pp. 11221131, 2013.

[4] J. A. Hutcheon, S. Lisonkova, and K. S. Joseph, "Epidemiology of pre-eclampsia and the other hypertensive disorders of pregnancy," Best Practice and Research: Clinical Obstetrics and Gynaecology, vol. 25, no. 4, pp. 391-403, 2011.

[5] National Institute for Health and Clinical Excellence, National Collaborating Centre for Women's and Children's Health (UK).
Antenatal Care: Routine Care for the Healthy Pregnant Woman. NICE Clinical Guidelines, No. 62., RCOG Press, London, UK, 2008.

[6] L. C. Y. Poon, N. A. Kametas, T. Chelemen, A. Leal, and K. H. Nicolaides, "Maternal risk factors for hypertensive disorders in pregnancy: a multivariate approach," Journal of Human Hypertension, vol. 24, no. 2, pp. 104-110, 2010.

[7] E. A. P. Steegers, P. Von Dadelszen, J. J. Duvekot, and R. Pijnenborg, "Pre-eclampsia," The Lancet, vol. 376, no. 9741, pp. 631644, 2010.

[8] IBM Corp, IBM SPSS Statistics for Windows, Version 21.0, IBM Corp, Armonk, NY, USA.

[9] L. C. Y. Poon, A. Syngelaki, R. Akolekar, J. Lai, and K. H. Nicolaides, "Combined screening for preeclampsia and small for gestational age at 11-13 weeks," Fetal Diagnosis and Therapy, vol. 33, no. 1, pp. 16-27, 2013.

[10] D. Wright, R. Akolekar, A. Syngelaki, L. C. Y. Poon, and K. H. Nicolaides, "A competing risks model in early screening for preeclampsia," Fetal Diagnosis and Therapy, vol. 32, no. 3, pp. 171-178, 2012.

[11] E. Scazzocchio, F. Figueras, F. Crispi et al., "Performance of a first-trimester screening of preeclampsia in a routine care lowrisk setting," American Journal of Obstetrics and Gynecology, vol. 208, no. 3, pp. 203.e1-203.e10, 2013.

[12] G. Di Lorenzo, M. Ceccarello, V. Cecotti et al., "First trimester maternal serum PIGF, free $\beta$-hCG, PAPP-A, PP-13, uterine artery Doppler and maternal history for the prediction of preeclampsia," Placenta, vol. 33, no. 6, pp. 495-501, 2012.

[13] M. Parra-Cordero, R. Rodrigo, P. Barja et al., "Prediction of early and late pre-eclampsia from maternal characteristics, uterine artery Doppler and markers of vasculogenesis during first trimester of pregnancy," Ultrasound in Obstetrics and Gynecology, vol. 41, no. 5, pp. 538-544, 2013.

[14] F. Crovetto, F. Crispi, E. Scazzocchio et al., "Performance of first trimester integrated screening for early and late small for gestational age newborns," Ultrasound in Obstetrics \& Gynecology, vol. 43, no. 1, pp. 34-40, 2014.

[15] R. Akolekar, A. Syngelaki, L. Poon, D. Wright, and K. H. Nicolaides, "Competing risks model in early screening for preeclampsia by biophysical and biochemical markers," Fetal Diagnosis and Therapy, vol. 33, no. 1, pp. 8-15, 2013.

[16] J.-M. Foidart, C. Munaut, F. Chantraine, R. Akolekar, and K. H. Nicolaides, "Maternal plasma soluble endoglin at 11-13 weeks' gestation in preeclampsia," Ultrasound in Obstetrics and Gynecology, vol. 35, no. 6, pp. 680-687, 2010.

[17] L. C. Y. Poon, N. A. Kametas, N. Maiz, R. Akolekar, and K. H. Nicolaides, "First-trimester prediction of hypertensive disorders in pregnancy," Hypertension, vol. 53, no. 5, pp. 812-818, 2009.

[18] L. C. Y. Poon, G. Karagiannis, I. Staboulidou, A. Shafiei, and K. $\mathrm{H}$. Nicolaides, "Reference range of birth weight with gestation and first-trimester prediction of small-for-gestation neonates," Prenatal Diagnosis, vol. 31, no. 1, pp. 58-65, 2011.

[19] G. Karagiannis, R. Akolekar, R. Sarquis, D. Wright, and K. H. Nicolaides, "Prediction of small-for-gestation neonates from biophysical and biochemical markers at 11-13 weeks," Fetal Diagnosis and Therapy, vol. 29, no. 2, pp. 148-154, 2011.

[20] K. Spencer, C. K. H. Yu, N. J. Cowans, C. Otigbah, and K. $\mathrm{H}$. Nicolaides, "Prediction of pregnancy complications by firsttrimester maternal serum PAPP-A and free $\beta$-hCG and with second-trimester uterine artery Doppler," Prenatal Diagnosis, vol. 25, no. 10, pp. 949-953, 2005. 
[21] A. Pilalis, A. P. Souka, P. Antsaklis et al., "Screening for preeclampsia and fetal growth restriction by uterine artery Doppler and PAPP-A at 11-14 weeks' gestation," Ultrasound in Obstetrics and Gynecology, vol. 29, no. 2, pp. 135-140, 2007.

[22] I. Cetin, B. Huppertz, G. Burton et al., "Pregenesys preeclampsia markers consensus meeting: what do we require from markers, risk assessment and model systems to tailor preventive strategies?” Placenta, vol. 32, supplement 1, pp. S4-S16, 2011. 


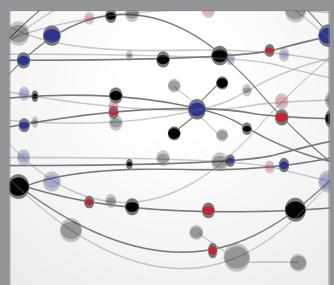

The Scientific World Journal
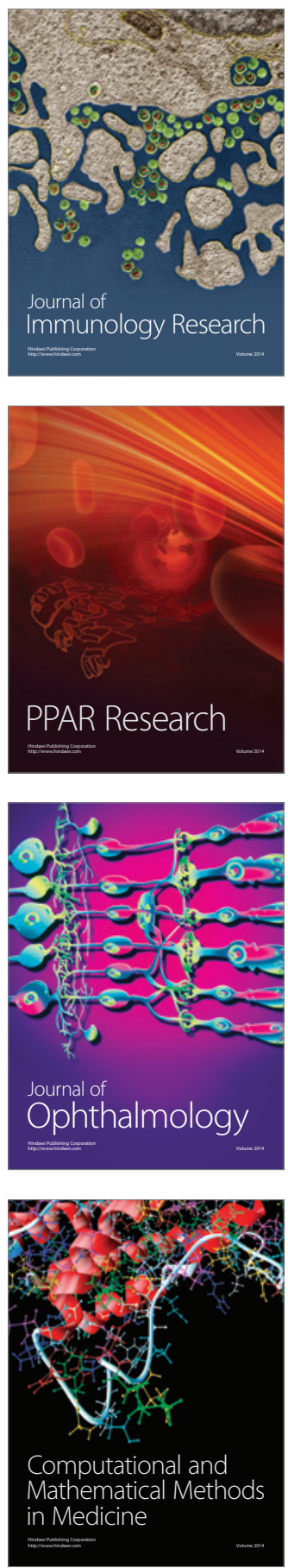

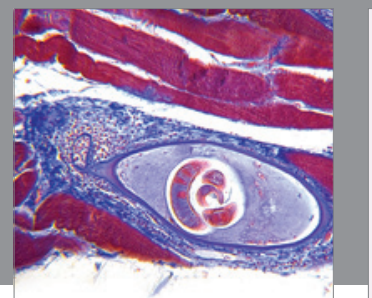

Gastroenterology

Research and Practice
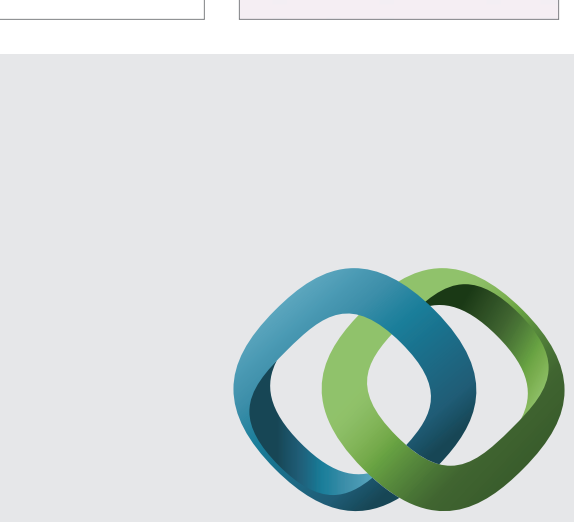

\section{Hindawi}

Submit your manuscripts at

http://www.hindawi.com
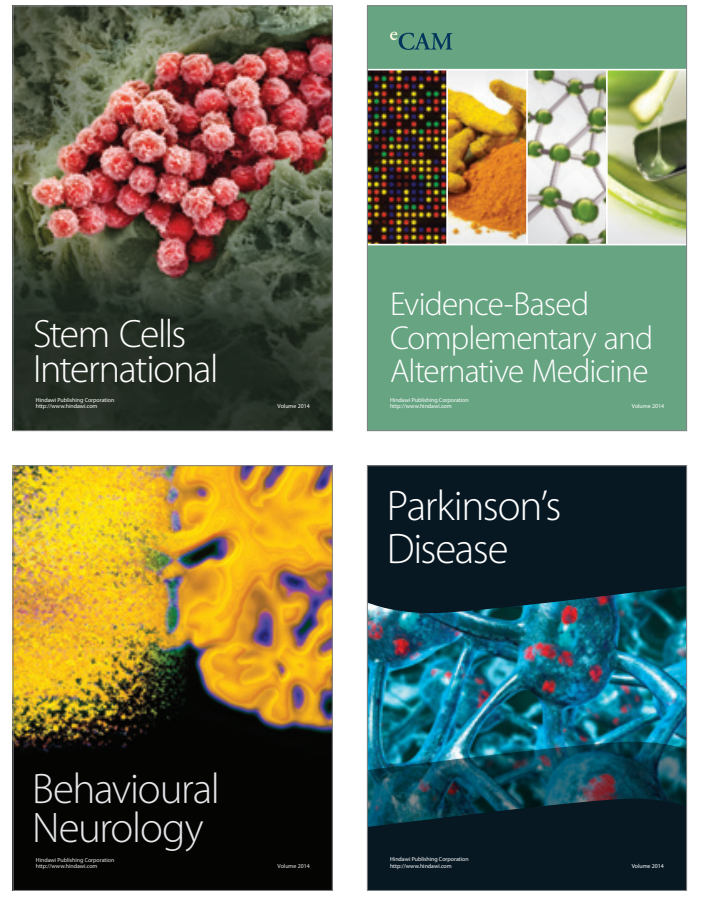
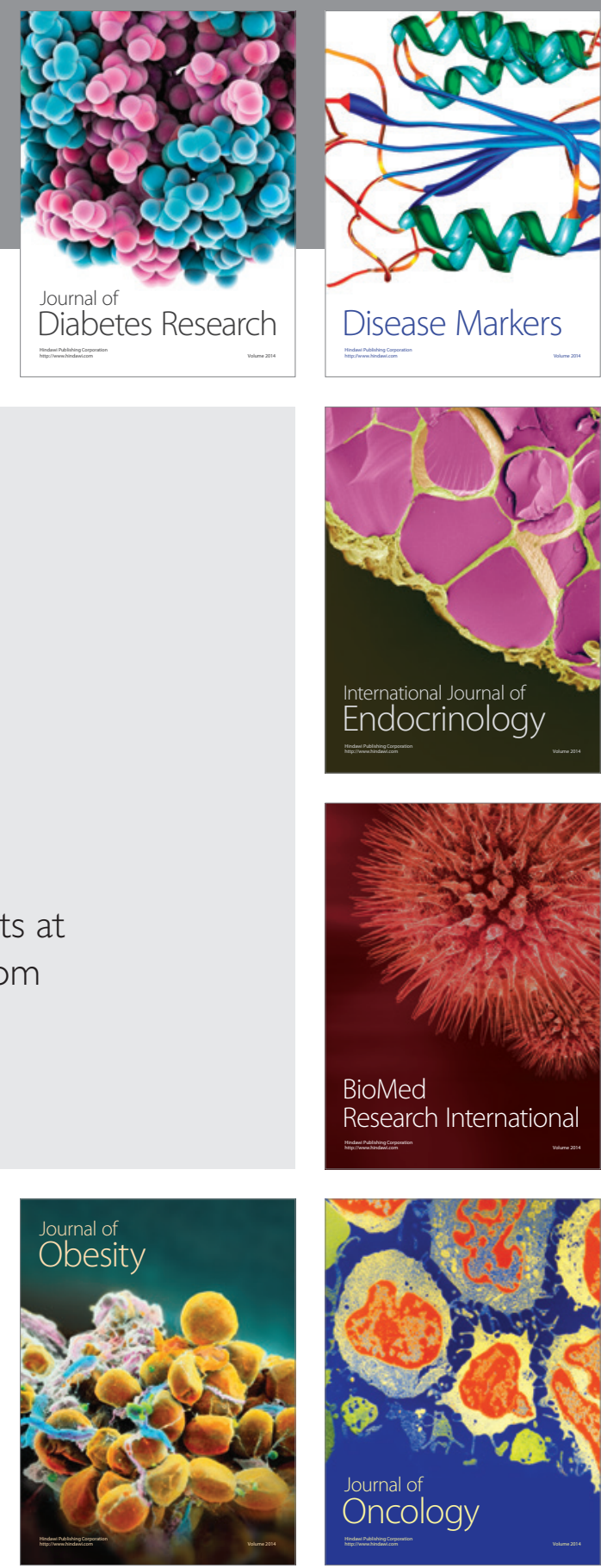

Disease Markers
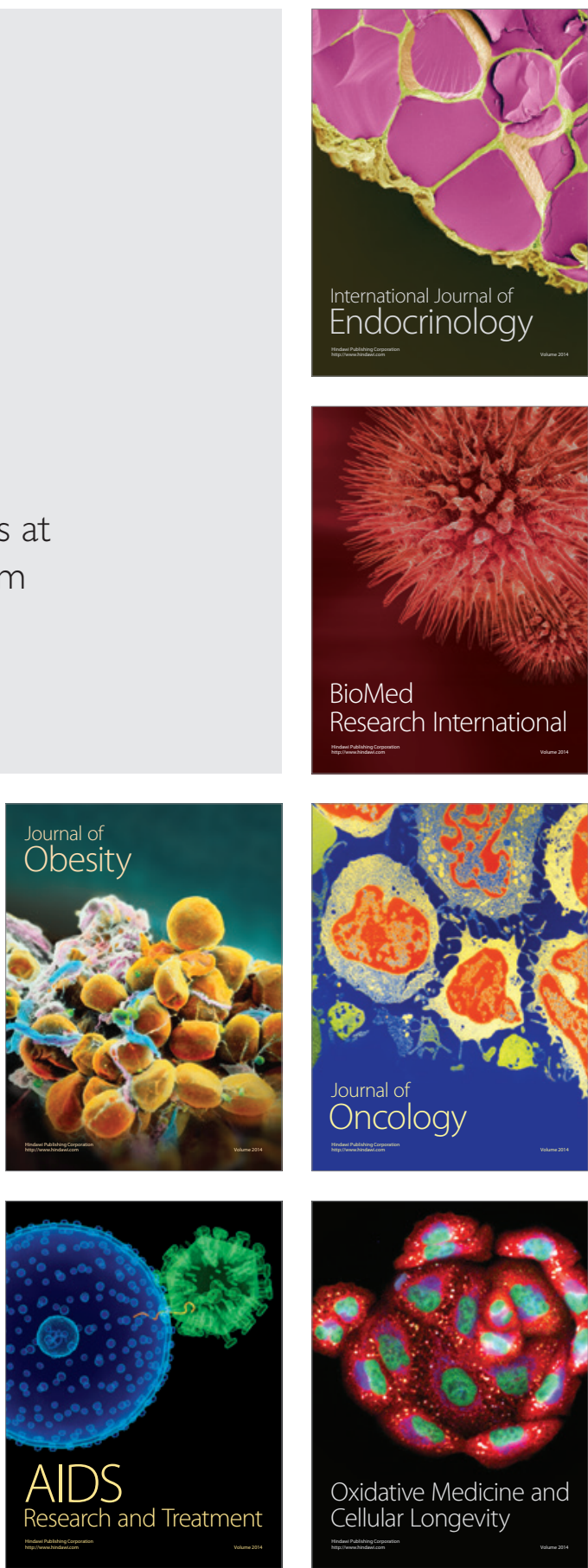